\title{
Diffraction and Spectroscopy: characterising thin ferromagnetic films from the local scale to the long-range... and beyond
}

\author{
I. Carlomagno ${ }^{1}$, J. Drnec ${ }^{2}$, R. Felici ${ }^{3}$, C. Meneghini ${ }^{4}$ \\ ${ }^{1}$ Elettra Sincrotrone Trieste, Italy \\ ${ }^{2} E S R F$, France \\ ${ }^{3}$ SPIN-CNR Roma, Italy \\ ${ }^{4}$ Università Roma Tre, Italy \\ ilaria.carlomagno@elettra.eu
}

The magnetic response of a system is the result of several contributions to the magnetic anisotropy which come from a plethora of effects. In the case of thin ferromagnetic films, the local-scale and long-range structural details, including film thickness, and interface interactions (intermixing, alloying, oxidation etc.) have a deep impact on the magnetism. Once known, these features can be used to tailor the magnetic response of the system, however, to fully control the system response, a deep knowledge is required.

To contrast the high reactivity of some ferromagnetic films, a passivating overlayer can be used: in these cases, further degrees of freedom add up in the definition of the magnetic response due to the upper interface phenomena. A deep understanding of such complex systems requires to assess and isolate the fine details linked to the interactions at the interfaces, and to those occurring within the film itself. Also, the oxidation prevention provided by the capping layer should be verified in view of application purposes.

Such a challenging task can be pursued only by combining complementary, state of the art techniques. This work presents the results of in-situ synchrotron radiation techniques and Magneto-Optic Kerr Effect (MOKE) measurements on $\mathrm{Gr} / \mathrm{Co} / \mathrm{Ir}$ systems, i.e. Co films intercalated between Graphene and $\operatorname{Ir}(111)$ [1]. The contributions to the in-plane and out-of-plane magnetic response of the system were evaluated based on Grazing Incidence X-Ray Diffraction (GI-XRD), X-ray Absorption Near Edge Spectroscopy (XANES), and Extended X-ray Absorption Fine Structure (EXAFS). The $\mathrm{Gr} / \mathrm{Co} / \mathrm{Ir}$ system is particularly interesting as the understanding of its magnetic behaviour requires to explore the thickness and thermal dependencies of local-scale and long-range anisotropies, to assess interface intermixing phenomena, and to evaluate the evolution of Co oxidation states (especially upon exposure to ambient conditions).

[1] I. Carlomagno et al., J. Appl. Phys. 120, 195302 (2016).

[2] J.Drnec, S. Vlaic, I. Carlomagno et al., Carbon 94, 554 (2015)

Keywords: Graphene, thin films, structure, magnetism 